\title{
Dual Pre-Motor Contribution to Songbird Syllable Variation
}

\author{
John A. Thompson, ${ }^{1,4}$ Mark J. Basista, ${ }^{1,4}$ Wei Wu, ${ }^{1,2}$ Richard Bertram, ${ }^{1,3}$ and Frank Johnson ${ }^{1,4}$ \\ ${ }^{1}$ Program in Neuroscience and Departments of ${ }^{2}$ Statistics, ${ }^{3}$ Mathematics, and ${ }^{4}$ Psychology, Florida State University, Tallahassee, Florida $32306-4301$
}

\begin{abstract}
Many forms of learning, including songbird vocal learning, rely on the brain's ability to use pre-motor variation and sensory feedback to guide behavior toward a specific target or goal. In the vocal control system of zebra finches (Taeniopygia guttata) the pre-motor mechanisms of vocal variation are thought to be vested primarily in a neural pathway that includes the basal ganglia. A second circuit that includes avian analogues of mammalian pre-motor and motor cortex (the vocal motor pathway) generates the patterned structure of learned adult song. Here, we tested the ability of the basal ganglia pathway to generate pre-motor vocal variation within the spectral and temporal dimensions of zebra finch song structure. In adult birds, ablation of the basal ganglia pathway significantly reduced the spectral and temporal dispersion of individual song syllables, with the exception of syllable pitch, where the reduction was not statistically significant when compared against surgical controls. We found a similar pattern of results using longitudinal comparisons (juvenile vs adult) to isolate the contribution of the basal ganglia pathway to spectral dispersion in populations of developing song syllablesvariation in syllable pitch was significantly smaller than in all other measured spectral features. The results indicate that pre-motor variation generated by the basal ganglia pathway may be sufficient to adjust vocal output toward highly acoustically dispersed targets of imitation, but suggest that complete acquisition of the pronounced variation in syllable pitch that characterizes adult song will necessitate a gradual developmental interaction between the basal ganglia and vocal motor pathways.
\end{abstract}

\section{Introduction}

Juvenile songbirds, like infant humans, learn to imitate adult vocal sounds. Behaviorally, this process begins with the production of unstructured and highly variable sequences of sounds known as "babbling." In juvenile male zebra finches the neural mechanisms of vocal babbling (subsong) are thought to be localized primarily within an anterior forebrain pathway (AFP) that includes the avian basal ganglia (Fig. 1) (Aronov et al., 2008). However, the AFP has roles beyond the control of vocal babbling as AFP influence on zebra finch song structure continues through the developmental period of vocal learning (Bottjer et al., 1984) and extends into adulthood (Brainard and Doupe, 2000).

The AFP intersects with a second pre-motor pathway [the vocal motor pathway (VMP)] that innervates the vocal musculature and drives adult zebra finch song (Simpson and Vicario, 1990). Figure 1 shows that the pre-motor region RA (robust nucleus of the arcopallium) provides a point of intersection between the dual pre-motor pathways, where individual RA neurons receive and integrate (Mooney and Konishi, 1991) synaptic input from HVC (proper name) and LMAN (lateral magnocellular nucleus of the nidopallium). Adult songs are therefore vocal composites produced by the integration of LMAN and HVC activity within RA (Fig. $1 B$ ). However, the relative influence of HVC and

Received Dec. 2, 2009; revised Sept. 26, 2010; accepted 0ct. 25, 2010

This work was supported by National Institutes of Health Grants DC008028 (J.A.T.) and DC02035 (F.J.). We thank Ross Henderson and Paul Hendrick for the design and fabrication of our songbird recording chambers and Joe Brazeau and Roy Miller for assistance in the analysis of vocal behavior. We thank Dr. Ofer Tchernichovski for use of the $\mathrm{SA}+$ software. The helpful advice of two reviewers is also acknowledged.

Correspondence should be addressed to Frank Johnson, Program in Neuroscience and Department of Psychology, Florida State University, Tallahassee, FL 32306-4301. E-mail: johnson@psy.fsu.edu. DOI:10.1523/JNEUROSCI.5967-09.2011

Copyright $\odot 2011$ the authors $\quad 0270-6474 / 11 / 310322-09 \$ 15.00 / 0$
LMAN on RA activity shifts during vocal development-LMAN activity largely determines the structure of juvenile subsong (Aronov et al., 2008) as HVC input to RA is initially quite sparse (Fig. 1A) (Mooney, 1992; Foster and Bottjer, 1998). During subsequent juvenile development, progressive and regressive anatomical changes in HVC and LMAN (Bottjer et al., 1985; Konishi and Akutagawa, 1985; Nordeen and Nordeen, 1988; Herrmann and Arnold, 1991; Johnson and Bottjer, 1992; Iyengar et al., 1999) result in an adult ratio of HVC:LMAN synapses on RA neurons of $\sim 2: 1$ (Fig. 1B) (Herrmann and Arnold, 1991).

While the AFP is not sufficient for normal song learning (e.g., a parallel basal ganglia pathway has recently been implicated in vocal development) (Bottjer and Altenau, 2010), the necessity of AFP activity for juvenile and adult vocal plasticity is well established (for review, see Brainard, 2004). What remains unclear is how AFP activity enables modification of vocal output. Hypotheses and supporting evidence encompass aspects of developmental critical periods, tutor song memory, instructive signaling, as well as pre-motor functionality (for review, see Mooney, 2009).

Here we focus on pre-motor aspects of AFP function, specifically the possibility that AFP pre-motor activity generates variation (vocal plasticity) within the temporal and spectral dimensions of zebra finch song structure, enabling goal-directed modification of vocal output toward a target of imitation (Tchernichovski et al., 2001). To test this possibility, song was measured before and after LMAN ablation in adult birds to ask whether the AFP can generate variation within multiple spectral (pitch, FM, entropy, pitch goodness) and temporal (syllable duration, intersyllable duration, motif duration) dimensions during the repeated production of individual song syllables (Fig. 1 $B, C$ ). We also made longitudi- 
A Subsong Circuitry
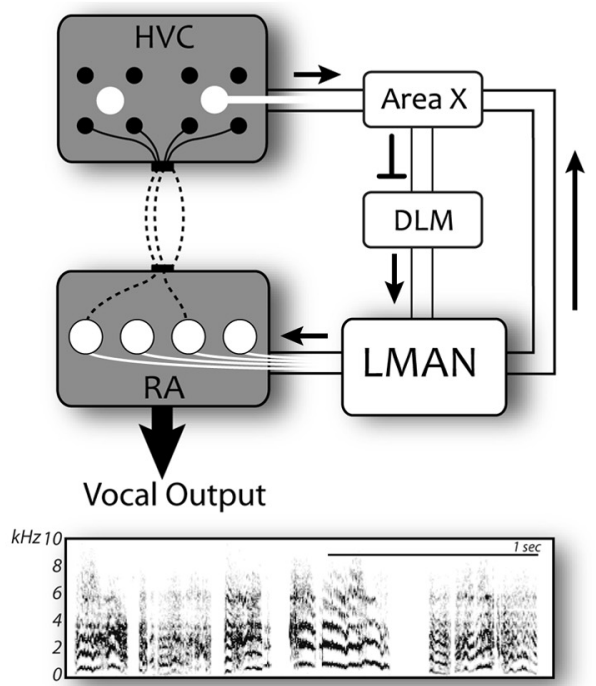

B Adult Song Circuitry
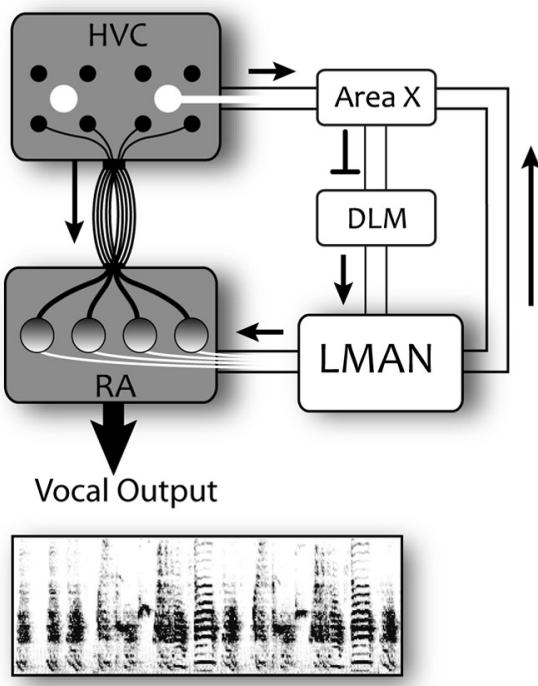

C LMAN Ablation Circuitry
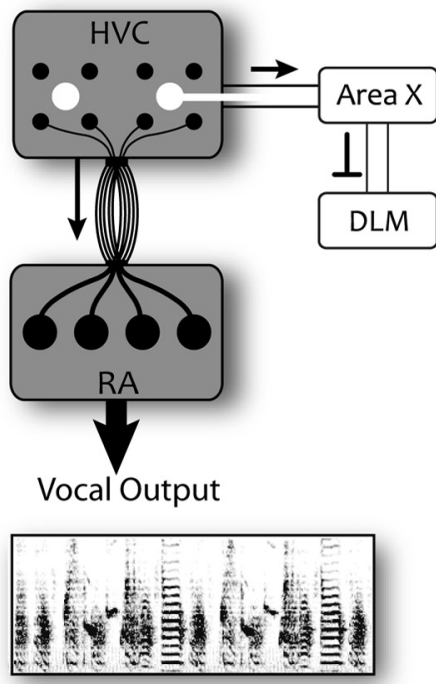

Figure 1. Schematics of the zebra finch song-control network show three circuit configurations with emphasis on input connectivity to the pre-motor nucleus RA. Frequency spectrograms show examples of the song behavior produced by each configuration. The VMP (black pathway) includes HVC, RA, and the hindbrain motor nuclei that control the vocal musculature. A second pre-motor pathway (the AFP, white pathway) originates in HVC and traverses basal ganglia (area X), thalamus (DLM, the medial dorsolateral nucleus of the thalamus) and LMAN before intersecting with the VMP at the level of RA. A, Vocal-motor development begins with subsong, a period of vocal babbling characterized by sequences of unstructured and unpatterned syllables. At this stage of juvenile development some HVC-RA neurons have established connectivity within RA (Mooney, 1992; Foster and Bottjer, 1998) although vocal output is driven primarily by LMAN (Aronov et al., 2008). $\boldsymbol{B}$, Learned adult song is highly patterned and driven primarily by the VMP. Adult song therefore involves a composite of pre-motor activity in the VMP and AFP. C, After LMAN ablation the VMP (and possibly other neural circuitry) (Bottjer and Altenau, 2010) drives patterned adult song.

nal comparisons of the range of spectral dispersion between populations of syllables produced during juvenile subsong (when song is unstructured and driven primarily by the AFP) and populations of syllables produced during the same bird's adult song (Fig. 1 $A, B$ ). This comparison allowed us to ask whether the AFP can generate variation that encompasses the spectral dimensions of adult song (as defined by pitch, FM, entropy, and pitch goodness).

\section{Materials and Methods}

\section{Animals and environment}

Male zebra finches were raised in our breeding colony or obtained from a commercial breeder. To compare vocal variation before and after LMAN ablation, adult birds were assigned randomly to an LMAN ablation group $(n=10)$ or a control surgery group $(n=10)$. To compare the spectral variation of juvenile subsong against adult song, birds raised in our breeding colony were assigned to a subsong/adult song group $(n=5)$. The validity of the spectral comparison in the subsong/adult song group depends in part on the finding by Aronov et al. (2008) that subsong is driven primarily by AFP activity. Therefore, we replicated a portion of their study (effects of HVC ablation on juvenile subsong, $n=4)$ and have included those results as supplemental Material 3 (available at www.jneurosci.org).

All birds were housed individually in medium-sized bird cages $(26.67 \times 19.05 \times 39.37 \mathrm{~cm})$ placed within computer-controlled environmental chambers $(75.69 \times 40.39 \times 47.24 \mathrm{~cm})$ that were fabricated by Florida State University Neuroscience Program engineering personnel. The design of the environmental chambers prevented visual as well as auditory access to other birds. A computer maintained both the photoperiod (14 h light, $10 \mathrm{~h}$ dark) and ambient temperature (set to $26^{\circ} \mathrm{C}$ ) within each chamber. Throughout the experiment, birds were provisioned daily with primarily millet-based assorted seed ( 400 Finch Blends, Jones Seed Company) and tap water. All daily care procedures and experimental manipulations of the birds were reviewed and approved by the Florida State University Animal Care and Use Committee.

\section{Surgeries}

LMAN ablation. Birds were deeply anesthetized with Equithesin $(0.04 \mathrm{cc})$ and then secured in a stereotaxic instrument. The skull was exposed by centrally incising the scalp and retracting the folds with curved forceps. Following application of avian saline $\left(0.75 \mathrm{~g}\right.$ of $\mathrm{NaCl} / 100 \mathrm{ml}$ of $\left.\mathrm{dH}_{2} \mathrm{O}\right)$ to the exposed area, small craniotomies were placed over the approximate location of LMAN bilaterally. To determine the location of LMAN, the bifurcation at the midsagittal sinus was used as stereotaxic zero. LMAN surgery involved eight penetrations, all of which had a depth of $2.6 \mathrm{~mm}$ : the first four were placed $3.8 \mathrm{~mm}$ anterior from zero, mirrored laterally at 1.4 and $1.9 \mathrm{~mm}$ from the midline. Two of the second four penetrations were placed more anterior $(5.4 \mathrm{~mm})$ from zero and positioned at $1.6 \mathrm{~mm}$ from the midline (which is midway between the first two lateral positions). The final two of the second four penetrations were more posterior $(2.2 \mathrm{~mm})$ from zero and again $1.6 \mathrm{~mm}$ lateral from the midline. The first four lesions were $3.5 \mathrm{~min}$ in duration and the second four were $2 \mathrm{~min}$ in duration. All eight lesions had a $100 \mu \mathrm{A}$ current. Following the surgery, the incision was treated with an antiseptic, sealed with veterinary adhesive, and the bird returned to its home cage.

LMAN control surgery. Birds were deeply anesthetized with Equithesin $(0.04 \mathrm{cc})$ and then secured in a stereotaxic instrument. Removal of two small cranial leaflets at $2.2 \mathrm{~mm}$ and $-2.2 \mathrm{~mm}$ lateral to the midsagittal sinus followed scalp incision and retraction. When surgery concluded, the incision was treated with an antiseptic, sealed with veterinary adhesive, and the bird returned to its home cage.

\section{Confirmation of LMAN ablation}

At the end of the 2 week postoperative period birds were given an overdose of Equithesin $(0.08 \mathrm{cc})$ and perfused intracardially with saline followed by ice-cold $4 \%$ paraformaldehyde. After overnight postfix by immersion in $4 \%$ paraformaldehyde, brains were sectioned coronally on a vibratome at $40 \mu \mathrm{m}$ thickness. Serial sections were then mounted onto microscope slides, thionin-stained, and coverslipped. The procedures used for lesion confirmation were similar to those described by Thompson et al. (2007). 
Analysis tools incorporated into Neurolucida software (MicroBrightField) were used to generate area estimates for serially traced contours of LMAN. Briefly, nonablated regions (i.e., area remaining) of LMAN, defined by Nissl stain, were traced and the areas summed and multiplied by section thickness. The remaining LMAN volume from each side was then divided by an average volume for intact unilateral LMAN $\left(0.11 \mathrm{~mm}^{3}\right.$, obtained from five intact birds) to estimate a percent unilateral LMAN remaining. Both unilateral estimates of remaining LMAN were then averaged to derive a value for total percent bilateral LMAN remaining.

\section{Song recording}

Birds were maintained in complete social isolation for the duration of the experiment. Therefore, only "undirected" songs (i.e., not directed toward a female) were recorded and analyzed. For all birds, song production was recorded in $24 \mathrm{~h}$ blocks using a unidirectional microphone placed facing the cage; the position of the microphone relative to the cage position remained unchanged throughout the experiment. Sounds transmitted by the microphone were monitored through a computer that was running sound-event triggered software (Avisoft Recorder, Avisoft Bioacoustics). Song was captured in bouts (2-7 s bursts of continuous singing during which the motif may be repeated $1-5$ times) and each song bout was saved as a time-stamped .wav file ( $44 \mathrm{kHz}$ sampling rate) onto the computer hard drive where each day of singing by each bird was saved under a single directory.

To ensure that we captured all song bouts, triggering settings for Avisoft Recorder were biased in favor of false-positive captures (i.e., .wav files that were comprised of repeated calls and/or cage noises). Therefore, for each bird and on each day of preoperative and postoperative singing, false-positive sound files were selectively deleted from each bird's database of .wav files. Detection and deletion of false-positive .wav files involved a three step process. First, we used Spectrogram 13.0 (Visualization Software) to convert sound files (.wav) into frequency spectrograph image files (.jpg). Second, we used an image-management program that contains an image-pattern recognition module (IMatch; Mario M. Westphal, Usingen, Germany) to identify .jpg files that contained singing-related spectra and deleted those images from the .jpg database. For the third and final step, we converted the file names of remaining false-positive images (i.e., .jpg files that did not contain singing-related spectra) from .jpg back to .wav and then batch-deleted those files from the main database of.wav files. Thus, each day of singing by each bird was reduced to a directory that contained only .wav files with song bouts. While each of these .wav files contained a song bout, some files also included cage noises (pecking on the cage floor, wing flaps or beak-wiping on the perch) and short and long calls that birds often produce in close temporal proximity to a song bout.

\section{Selection of songs for analysis}

LMAN ablation and control surgery. Postoperative singing was monitored every day, beginning with the first day after surgery through the day of perfusion. Following LMAN ablation, birds took on average $2.7 \mathrm{~d}( \pm 0.4$, $\mathrm{SE})$ to begin postoperative singing, whereas singing returned $1.2 \mathrm{~d}( \pm 0.4$, SE) following control surgery.

Although birds were recorded continuously during the 3 week postoperative period, not all days were used for analysis: we used the first $3 \mathrm{~d}$ of postoperative singing in which birds achieved $75 \%$ of their preoperative daily song bout production, and then selected 1-4 additional days of singing produced during the remainder of the postoperative period. For most birds ( 12 of 20) this resulted in the analysis of $7 \mathrm{~d}$ each of preoperative and postoperative singing, although for 3 birds in the LMAN ablation group and for 5 birds in the control surgery group the average number of compared days of singing was 5 (range 4-6). On each of these days, the number of song bouts analyzed per bird was normalized to the bird that produced the lowest number of song bouts in each group. On average, this resulted in the measurement of 5481 preoperative and 5624 postoperative syllables per bird in the LMAN ablation group, and 5574 preoperative and 5724 postoperative syllables per bird in the control surgery group. Finally, in a preliminary analysis we found that changes in syllable variation following LMAN ablation were evident on the first day of postoperative singing and relatively stable thereafter (as reported previously by Kao and Brainard, 2006). Therefore, in all subsequent statis- tical analyses singing is characterized simply as "preoperative" or "postoperative."

Subsong/adult song. To compare subsong against adult song, $n=5$ juvenile males were removed from the breeding colony at $35 \mathrm{~d}$ posthatch, placed in sound recording chambers, and recorded until adulthood (120 $\mathrm{d}$ posthatch). In these birds, subsong captured at the onset of song development (between 35 and $42 \mathrm{~d}$ posthatch) was compared with their own adult song generated at $120 \mathrm{~d}$ posthatch. We analyzed 40 song bouts for each bird: 20 bouts of subsong and 20 bouts of adult song. Following placement in a recording chamber at $35 \mathrm{~d}$ posthatch, juvenile birds initially generate a low number of song bouts (Johnson et al., 2002). Therefore, days of singing that contained $<20$ bouts of song were excluded, and the first day of singing in which a juvenile generated $20+$ bouts of subsong was selected for analysis-in all cases this occurred before $42 \mathrm{~d}$ posthatch, at which time the singing of each bird lacked pure tones and showed no discernable repetition of syllable types (Fig. 1 A, see spectrogram of subsong). At this stage of development, the spectral structure of subsong is determined primarily by the AFP (Aronov et al., 2008) (supplemental Material 3, available at www.jneurosci.org). For each bird, we randomly selected 20 bouts of subsong and 20 bouts of adult song (recorded at $120 \mathrm{~d}$ posthatch) for analysis. On average, this resulted in the measurement of 218 juvenile subsong syllables and 329 adult song syllables per bird.

\section{Measurement of spectral variation in the production of individual} syllables before and after LMAN ablation in adult birds

Previous investigation of spectral variation in zebra finch song patterns has focused on pitch (Kao et al., 2005; Kao and Brainard, 2006). In these studies, pitch values are obtained by measuring the fundamental frequency (F0) of 50-200 ms segments of harmonic syllables that lack frequency modulation (FM). Selection of these unmodulated harmonic syllable segments is manual, effectively limiting the number of segments that are measured per condition in each bird (e.g., 16-90 segments per condition reported by Kao and Brainard, 2006).

To expand the number of syllable types and spectral features measured, we used Sound Analysis Pro software (SA+ version 1.04a) (Tchernichovski et al., 2000) to automate (following a manual thresholding step, see below) measurement of spectral variation in whole syllables. In addition to average syllable values for pitch (a measure of the frequency structure of sound), average syllable values were obtained for FM (a measure of change in the temporal structure of sound), entropy (a measure of the nonharmonicity of sound), and pitch goodness (a measure of the harmonic structure of sound). Pitch, FM, entropy, and pitch goodness were selected because songbirds demonstrate the ability to make behavioral discriminations in response to variation in all four of these spectral features [pitch (Cynx and Shapiro, 1986), FM (Lohr et al., 2006), entropy (Lohr and Dooling, 1998), and pitch goodness (Cynx et al., 1990)].

Zebra finch song syllables contain few pure tones (where assessment of pitch is unambiguous) and many zebra finch syllables are composites of frequency-modulated harmonic and nonharmonic sound (although the nonharmonic sounds incorporated into zebra finch songs generally show evidence of bandpassing). Measurement of syllable pitch in zebra finches therefore requires two different acoustic measures (Tchernichovski et al., 2000). While pitch can be reasonably estimated when sound is harmonic - it is related to the F0 of the sound-nonharmonic, noisy sounds possess pitch only if effectively bandpassed in frequency and/or amplitude. In these instances the mean frequency (MF) of nonharmonic sounds provides a practical estimate of the pitch of the sound. Thus, SA+ uses a measure of pitch goodness to distinguish between harmonic and nonharmonic sound and then uses F0 or MF (respectively) to estimate pitch. This was done here using an FFT window size of $9.27 \mathrm{~ms}$ and a window advance of $1.36 \mathrm{~ms}$ (i.e., default settings for zebra finches in $\mathrm{SA}+$ ).

Syllable-threshold controls in SA+ (amplitude, entropy, minimum syllable and minimum stop duration) were manually configured for the motif of each individual bird so that the software reliably identified each syllable in the bird's motif. Once established, threshold settings for each bird were noted and applied without change to all song bouts produced 
in all preoperative and postoperative days of singing, insuring valid pre/ postcomparisons of phonology. Scatter plots were used to verify that threshold settings had been accurately specified-for these plots, syllable duration was always plotted on the $x$-axis and one of the measured spectral features (pitch, FM, entropy, pitch goodness) was plotted on the $y$-axis. Within these plots, each data point represents an individual syllable and, thus, clusters of data points signify repeated production of a specific song syllable. Clusters observed within the scatter plots were then compared against a frequency spectrograph of the bird's motif to confirm that each syllable in the bird's motif was represented by a discrete cluster of data points (for additional details, see Wu et al., 2008).

To identify syllables across all days of singing for each bird, we imported the SA+ data into Matlab (MathWorks Inc.). Within Matlab, all days of singing for each bird were converted into a single "master" scatter plot using duration and one spectral feature-the spectral feature used was the one that produced the most distinct spatial clustering of syllables (which varied between birds, depending on the acoustic structure of syllables in a bird's motif). Then within this master scatter plot (which included syllables from all days of singing), polygonal boundaries were traced around each syllable to create a "syllable template" that reliably captured all preoperative and postoperative iterations of each syllable in each bird's motif (see supplemental Material 1, supplemental Fig. S1-1, available at www.jneurosci.org as supplemental material). Using this method, we identified $n=45$ syllable clusters in the LMAN ablation group and $n=38$ syllable clusters in the control surgery group. Introductory syllables were not included in this analysis.

After calculating the median value of each syllable cluster, the interquartile range (IQR) (the range between the first and third quartile) was used to assess variation in pitch, FM, entropy, and pitch goodness. We selected IQR because it was demonstrably robust to the presence of small numbers of outlying syllable values that were sometimes associated with syllable clusters. Although these outlying values never accounted for $>1 \%$ of the total syllable values in a cluster, we found that the more commonly used CV (coefficient of variation) was sometimes considerably distorted by a small number of outlying syllable values. Supplemental Material 1 (available at www.jneurosci.org) presents a comparison of the sensitivity of CV and IQR to outlying syllable values. Thus, given the structure of our dataset, IQR provided the most appropriate and robust estimate of dispersion. Although IQR retains units of measure, we also generated normalized IQR values for each spectral feature $\left[\left(\mathrm{IQR}_{\text {pre }}-\right.\right.$ $\left.\left.\mathrm{IQR}_{\text {post }}\right) / \mathrm{IQR}_{\text {pre }}\right]$. This step enabled statistical comparison between birds that received LMAN ablation and birds that received control surgery.

\section{Measurement of temporal variation in the production of}

individual syllables before and after LMAN ablation in adult birds We analyzed the temporal variation of song at three levels: syllable duration, intersyllable duration and motif duration. For each bird we selected $n=50$ motifs from $1 \mathrm{~d}$ of preoperative singing (last day before surgery) and $n=50$ motifs from $1 \mathrm{~d}$ of postoperative singing ( $>1$ week postsurgery). All motifs were analyzed using the Explore and Score module in $\mathrm{SA}+$, which generates measures of the temporal onset and duration of each syllable.

Syllable thresholds in SA+ were set to reliably partition motifs into whole syllables to generate values for the duration of syllables $(n=45$ for the LMAN ablation group, $n=38$ for the control surgery group), intersyllable intervals ( $n=35$ for the LMAN ablation group, $n=30$ for the surgery control group), and motifs ( $n=10$ in LMAN ablation and surgery control groups). Median and IQR values for each syllable, intersyllable and motif duration were then calculated.

\section{Longitudinal comparison of spectral variation in syllable populations}

A heterogeneous population of unstructured and undifferentiated syllables typifies juvenile subsong (Fig. $1 \mathrm{~A}$, see spectrogram). Thus, during subsong the overall absence of patterned sequences of identifiable syllable types precludes measurement of temporal and spectral variation in repeated instances of the same syllable (as in the analysis described above for adult birds). Therefore, to compare spectral variation produced during subsong to that produced during adult song we analyzed all subsong syllables and all adult syllables as single populations. This approach allows quantification of the overall width of syllable feature distributions across vocal development in individual birds, and comparison of the spectral variation produced when song is driven primarily by the AFP (subsong) to the spectral variation produced by the integrated activity of the vocal circuitry as a whole (adult song). This comparison allows us to ask whether the AFP can generate variation that encompasses the spectral dimensions of adult song (as defined by pitch, FM, entropy, and pitch goodness).

$\mathrm{SA}+$ was used to partition bouts into syllables and measure the spectral quality of syllables based on pitch, FM, entropy, and pitch goodness. We assessed feature variation across all syllables for each bird during subsong and adult song by calculating the IQR for each spectral feature, analyzing all syllables as a single group. IQR was used due to the presence of outliers in the syllable populations (which were particularly evident in subsong syllable populations). We also generated normalized IQR values $\left[\left(\mathrm{IQR}_{\text {subsong }}-\mathrm{IQR}_{\text {adult song }}\right) / \mathrm{IQR}_{\text {subsong }}\right]$ to compare the magnitude of developmental change in variation across different spectral features.

\section{Statistics}

LMAN ablation and control surgery. The dispersion (IQR) and central tendency (median) of all measured spectral and temporal features were analyzed using within-subject (pre - post) comparisons (Wilcoxon matched-pairs signed-rank, Prism 5.03, GraphPad Software). Although a previous study of the effect of LMAN ablation on syllable pitch used paired sign tests to analyze data (Kao and Brainard, 2006), we wanted to consider the magnitude of change (in addition to the direction of change) when interpreting the effect of LMAN ablation. The dispersion (IQR) of spectral features was also analyzed using between-subject (LMAN ablation vs control surgery) comparisons (Mann-Whitney) (Prism 5.03, GraphPad Software). Normalized IQR values [( $\left.\left.\mathrm{IQR}_{\text {pre }}-\mathrm{IQR}_{\mathrm{post}}\right) / \mathrm{IQR}_{\text {pre }}\right]$ were used for these comparisons. Results of all within-subject statistical tests (which included testing with CV values for purposes of comparison) are presented in supplemental Material 2 (spectral comparisons, supplemental Tables 1 and 2; temporal comparisons, supplemental Tables 3 and 4, available at www.jneurosci.org as supplemental material).

Subsong/adult song. For each spectral feature, within-subject comparisons were made between IQR values obtained during subsong and during adult song (Wilcoxon matched-pairs signed-rank test). To compare developmental change in variation across spectral features, normalized $\mathrm{IQR}$ values $\left[\left(\mathrm{IQR}_{\text {subsong }}-\mathrm{IQR}_{\text {adult song }}\right) / \mathrm{IQR}_{\text {subsong }}\right]$ for pitch, $\mathrm{FM}$, entropy and pitch goodness were analyzed with a one-way ANOVA (Prism 5.03, GraphPad Software).

\section{Results}

\section{Amount of remaining LMAN following ablation surgery}

The extent of LMAN ablation in all adult birds exceeded the $>70 \%$ value established by Kao et al. (2005) as sufficient to abolish the behavioral influence of LMAN on the song of adult male zebra finches. On average, the remaining bilateral volume of LMAN was close to $2 \%$ (the largest remaining LMAN volume was $17 \%)$. As a check on the bilateral symmetry of the LMAN ablations, we compared left and right hemisphere estimates of percent remaining LMAN and found that they were not significantly different $\left(t\right.$ test, $\mathrm{t}_{9}=1.34 ; p=0.214$ ).

\section{Effect of LMAN ablation on spectral variation during repeated production of individual syllables}

Figure $2 \mathrm{~A}$ shows column scatter plots (each data point is one syllable) of difference scores for postoperative variation in syllable pitch, FM, entropy, and pitch goodness for adult birds that received LMAN ablation ( $n=45$ syllables, black data points) or control surgery ( $n=38$ syllables, gray data points). Positive syllable values indicate less postoperative variation whereas negative syllable values indicate more postoperative variation. Birds with LMAN ablation showed significant postoperative reductions in 
variation for syllable pitch (Wilcoxon signed-rank, $p<0.01)$, FM ( $p<0.01)$, entropy $(p<0.01)$ and pitch goodness $(p<0.01)$.

However, while birds with control surgery showed no significant postoperative change in variation for syllable FM ( $p=$ 0.36 ), entropy ( $p=0.45$ ), or pitch goodness $(p=0.27)$, control surgery produced a near-significant trend toward reduced postoperative variation for syllable pitch $(p=0.051, p=0.017$ if a paired sign test is used) (cf. Kao and Brainard, 2006). This result suggests that surgery experience specifically reduces postoperative variation in syllable pitch, although the average reduction following LMAN ablation was larger ( $2 \%$ reduction following control surgery, $8.9 \%$ reduction following LMAN ablation).

We then compared birds with LMAN ablation to birds that received control surgery to determine whether the reduction in pitch variation differed significantly between the two groups-normalized IQR values were used for this comparison $\left[\left(\mathrm{IQR}_{\text {pre }}-\mathrm{IQR}_{\text {post }}\right) / \mathrm{IQR}_{\text {pre }}\right]$. Figure 3 shows that the postoperative reduction in variation for syllable pitch did not differ between birds with LMAN ablation and birds with control surgery (Mann-Whitney, $p=$ $0.388)$. However, when compared with controls, LMAN ablation did produce a significant reduction in postoperative variation in all other measured spectral features (FM, $p=0.002$; entropy, $p=0.038$; pitch goodness, $p=0.006$; data not shown). These statistical comparisons show that while LMAN ablation significantly reduces variation in the FM, entropy, and pitch goodness of adult song syllables, the postoperative decrease in pitch variation that we observed following LMAN ablation cannot be attributed solely to ablation of LMAN.

The central tendency of syllable spectral features was also analyzed for an influence of LMAN ablation or control surgery (data not shown). LMAN ablation did not have a significant effect on median values for syllable pitch (Wilcoxon signed-rank, $p=$ $0.78)$, FM $(p=0.11)$, entropy $(p=0.67)$, or pitch goodness $(p=0.75)$ and we obtained the same pattern of results for birds with control surgery (pitch, $p=0.81 ; \mathrm{FM}, p=0.46$; entropy, $p=$ 0.45 ; pitch goodness, $p=0.84$ ). While there is microstimulation evidence that LMAN activity can alter the central tendency of pitch (Kao et al., 2005) selective stimulation of dorsal (lower pitch) versus ventral (high pitch) regions of LMAN was necessary to observe shifts in central tendency. It seems likely that dorsal versus ventral aspects of LMAN would have to be selectively targeted to observe a similar shift in the central tendency of syllable spectral features following ablation.

Thus, under our experimental conditions the contribution of LMAN to the spectral structure of adult song syllables is specifically one of expanding the range of spectral dispersion generated by the remainder of the vocal circuitry and the vocal musculature-complete LMAN ablation does not alter the central tendency of syllable spectral values, although the work of Kao et al. (2005) suggests that partial ablation (dorsal vs ventral) might reveal such an effect. Another caveat is the contribution of LMAN to variation in syllable pitch. Because birds with control surgery also showed a trend toward postoperative reduction in variation for syllable pitch, the magnitude of the LMAN contribution to variation in syllable pitch is not statistically larger than, or additive to, the effect produced by control surgery alone.

\section{Effect of LMAN ablation on temporal variation during repeated production of individual syllables}

Figure $2 B$ shows column scatter plots of difference scores for postoperative variation in the temporal structure of adult song (syllable duration, intersyllable duration, and motif duration). Positive syllable values indicate less postoperative variation whereas negative syllable values indicate more postoperative variation. Birds with LMAN ablation showed significant postoperative reductions in variation for syllable duration (Wilcoxon signed-rank, $p<0.01)$ and intersyllable duration $(p<0.01)$, whereas an apparent trend toward decreased variation in motif duration did not reach statistical significance ( $p=0.11)$. In contrast to the effects of LMAN ablation, control surgery produced a trend toward increased variation in all three temporal measures (particularly for syllable duration), although none were statistically significant (syllable duration, $p=0.06$; intersyllable duration, $p=0.13$; motif duration, $p=0.16$ ).

Median values for syllable duration and intersyllable duration were also influenced by LMAN ablation, but not control surgery. Figure 4 shows column scatter plots of difference scores for median syllable duration and median intersyllable duration, with 


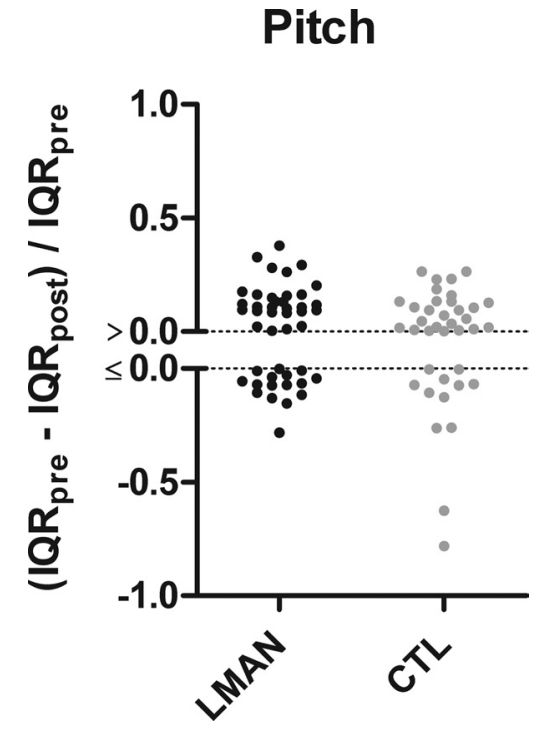

Figure 3. Column scatter plots (each data point is one syllable) of normalized IQR values indicate that the effect of LMAN ablation ( $n=45$ syllables, black data points) on the dispersion of syllable pitch is not statistically different from the effect of control surgery ( $n=38$ syllables, gray data points). Between-subject test, Mann-Whitney, $p=0.388$.

upward shifts indicating a shortening of postoperative duration, and downward shifts indicating a lengthening of postoperative duration. LMAN ablation produced a shortening of median syllable durations (Wilcoxon signed-rank, $p<0.01$ ) and a lengthening of median intersyllable durations $(p<0.05)$. Due to these contrasting effects of LMAN ablation on median syllable duration (shorter) and median intersyllable duration (longer), there was no effect of LMAN ablation on median motif duration ( $p=0.32$, motif duration being a summing of all syllable and intersyllable durations in a bird's motif). Control surgery did not significantly affect median values for any temporal feature, although there was a trend for longer syllable durations (syllable duration, $p=0.10$; intersyllable duration, $p=0.90$; motif duration, $p=0.28)$.

Overall, measurement of the temporal structure of adult song syllables indicates that LMAN activity expands the range of temporal dispersion generated by the remainder of the vocal circuitry and the vocal musculature. However, LMAN activity also affected the central tendency of syllable and intersyllable durations, acting to lengthen the duration of individual song syllables, but not the motif, thereby resulting in shorter intersyllable durations.

\section{Longitudinal comparison of spectral dispersion in syllable populations}

Here we compared the range of dispersion in the population distribution of each syllable spectral feature during subsong (when singing is generated primarily by the AFP) to the range of dispersion present in the learned adult song. Our rationale was based in part on the findings of Aronov et al. (2008), who reported that juvenile subsong was driven by the AFP. Because these findings are central to the design of our experiment, we replicated a portion of the Aronov et al. (2008) study, measuring the spectral characteristics of subsong before and after HVC ablation in a group of juvenile male zebra finches $(n=4$, included here as supplemental Material 3, available at www.jneurosci.org). HVC ablation removes input to RA as well as area X of the AFP (Fig. 1). Therefore, this manipulation tests whether the remaining structures of the AFP (LMAN, area X, DLM, and RA) are
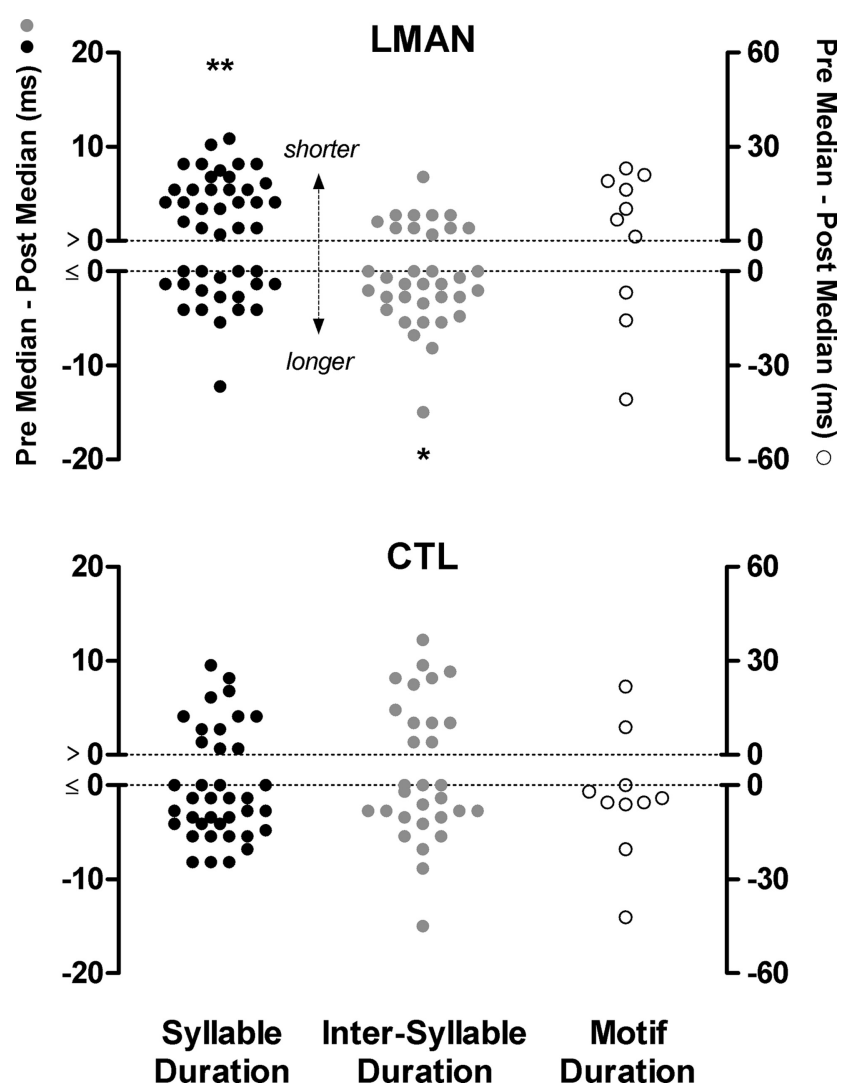

Figure 4. Column scatter plots (each data point is one duration measure) show difference scores for postoperative change in the central tendency (median) of syllable duration (black data points), intersyllable duration (gray data points), and motif duration (clear data points). Positive values indicate shorter postoperative durations whereas negative values indicate longer postoperative durations. Stars indicate significant postoperative change in median duration (Wilcoxon signed-rank tests). LMAN ablation produced a significant postoperative shortening of syllable duration ( $n=45, p<0.01$ ), but a significant postoperative lengthening of intersyllable duration ( $n=35, p<0.05$ ). As a result of these contrasting effects, LMAN ablation did not have a significant effect on motif duration $(n=10)$. Control surgery did not produce a significant effect on syllable duration $(n=38)$, intersyllable duration $(n=30)$, or motif duration $(n=10)$

sufficient to generate the spectral characteristics of subsong. No statistically significant effects were found for syllable FM, entropy, or pitch goodness, but a small effect of HVC ablation on the pitch of subsong syllables was noted (see supplemental Fig. S3-1, available at www.jneurosci.org as supplemental material). That is, the mean pitch of subsong syllables was slightly reduced $(8 \%)$ following HVC ablation (paired $t$ test, $\mathrm{t}_{3}=3.38, p=0.04$ ). However, comparison of syllable scatter plots of juvenile singing before and after HVC ablation (see supplemental Fig. S3-2, available at www.jneurosci.org as supplemental material) confirm the finding of Aronov et al. (2008) that AFP activity determines the spectral characteristics of subsong (although it should be noted that this conclusion is limited to the spectral characteristics we have measured here-pitch, FM, entropy, pitch goodness). Therefore, we compare singing driven primarily by the AFP (subsong) against singing driven by the integrated activity of the complete vocal circuitry (adult song).

Figure $5 A-D$ shows scatter plot comparisons of the juvenile subsong syllables (black data points) against the adult song syllables (gray data points) of a representative bird (A, pitch, B, FM, C, entropy, $\mathrm{D}$, pitch goodness; spectral feature on the $y$-axis over syllable duration on the $x$-axis). These visual comparisons (which 

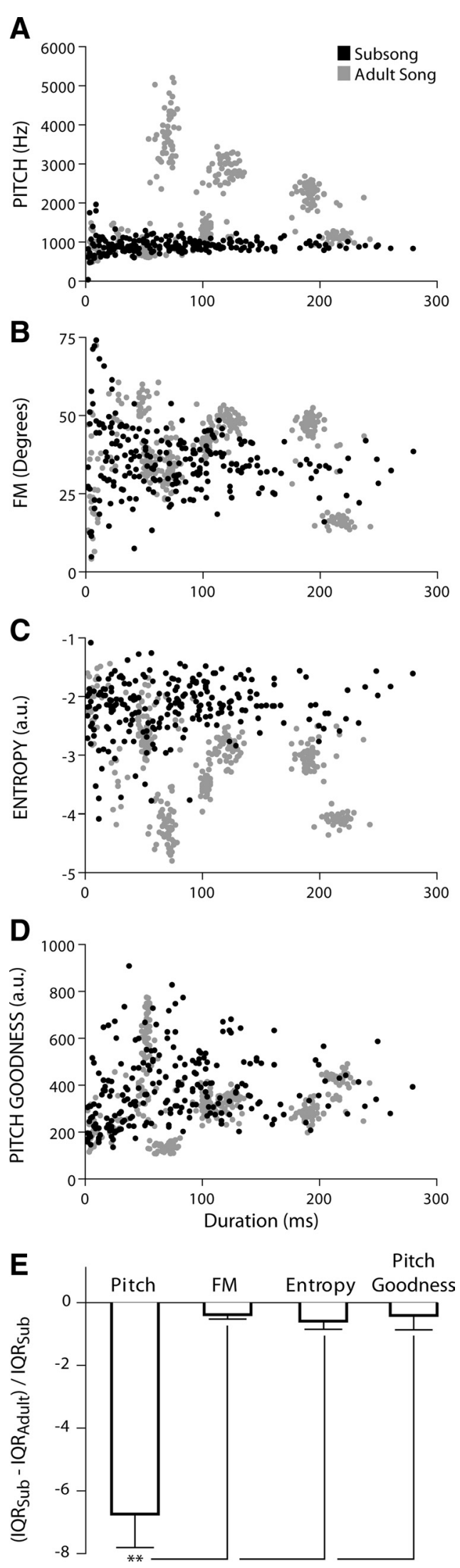

Figure 5. Only variation in the pitch of song syllables differs between juvenile subsong and adult song. $\boldsymbol{A}-\boldsymbol{D}$, Scatter plots of syllable duration by spectral feature $(\boldsymbol{A}$, pitch; $\boldsymbol{B}$, FM; $\boldsymbol{C}$, entropy; $\boldsymbol{D}$, pitch goodness) from a representative bird show that dispersion of syllable values for pitch is limited during subsong (black) when compared with adult song (gray) —all other features show similar variation during subsong and adult song. $\boldsymbol{E}$, The show the overall width of the population distribution of syllable spectral features) demonstrate that juvenile subsong displayed significantly less variation in syllable pitch than adult song (this is also evident in the frequency spectrograms of subsong and adult song shown in Fig. 1; only the adult song contains syllable segments with F0s in the $3-5 \mathrm{kHz}$ range). In contrast, the range of variation present in the distribution of all other syllable spectral features was roughly equivalent between subsong and adult song. Interestingly, if AFP-generated variation in syllable spectral features were simply random, one might expect the dispersion of syllable spectral values to be significantly wider or more expansive during subsong. One possibility is that early tutoring experience biases the range of AFP-generated variation in syllable FM, entropy, and pitch goodness toward specific acoustic targets defined by the tutor song.

Statistical tests of IQR difference scores (subsong - adult song) for each spectral feature confirmed that only pitch showed a significant increase in variation across vocal development (Wilcoxon signed-rank, $p<0.05$ ). These findings are similar to the developmental increase in pitch variation recently observed by Bottjer and Altenau (2010), although our subsong recordings appear to have been made at an earlier age. Similarly, a one-way ANOVA of normalized IQR values revealed that the developmental increase in variation for pitch was significantly larger than that for FM, entropy, or pitch goodness $\left(F_{(3,19)}=27.56\right.$; $p<$ 0.0001) (Fig. 5E).

We did not specifically measure the temporal properties of juvenile subsong (the lack of repeated syllable types in subsong precludes such an analysis), but substantial variation in the duration of subsong syllables is clearly evident in frequency spectrograms of subsong (Fig. 1A) and in spectral feature $\times$ duration scatter plots of song (Fig. $5 A-D$, black data points). Together, these data and the results from adult birds with LMAN ablation (above) indicate that the AFP contributes variation to the temporal structure of song in juvenile and adult birds.

\section{Discussion}

By measuring adult song before and after LMAN ablation we found that the AFP shapes the structure of adult song syllables by increasing the spectral dispersion of sounds generated by the remaining vocal circuitry and vocal musculature. Analysis of syllable temporal structure showed that LMAN ablation alters both the dispersion and central tendency of syllable and intersyllable duration. However, while we found that AFP activity lengthens syllable durations, we observed a compensatory shortening of intersyllable durations, meaning that AFP activity did not influence the central tendency of motif duration. AFP activity appears to influence the duration of local song elements (syllable, intersyllable durations) but the global duration of the patterned sequence of sounds that comprises the motif is determined elsewhere within the vocal circuitry.

Our analysis of syllable pitch provides an exception to the view that AFP activity increases spectral dispersion in the structure of adult song syllables. Although we found a significant reduction in pitch variation following LMAN ablation, a near significant ( $p=$ $0.051)$ reduction in pitch variation also occurred in birds that received control surgery. This was not a general effect of control surgery as the reduction in pitch variation was the only spectral or

$\leftarrow$

developmental increase in variance (IQR) for the pitch of song syllables is significantly larger than that for FM, entropy, or pitch goodness ( $p<0.0001)$. 
temporal measure where control surgery produced a trend that was similar to the effects of LMAN ablation. Moreover, pitch was the only spectral feature where statistical comparison against controls failed to find significantly less variation in birds that received LMAN ablation. The selective sensitivity of pitch variation to control surgery may indicate that manipulations associated with surgery dampen sources of pitch variation that lie outside of LMAN or the AFP, thereby obscuring any reduction in pitch variation associated with ablation of LMAN. Thus, under our experimental conditions the postoperative decrease in pitch variation that we observed following LMAN ablation cannot be attributed solely to ablation of LMAN.

\section{Relationship to previous findings}

A previous report by Kao and Brainard (2006) provides a contrast to our findings. These investigators found that LMAN ablation produced a significant reduction in pitch variation. While we used identical surgical anesthesia and ablation techniques, differences in the approach to pitch measurement, statistical analysis, and experimental design could explain the different experimental outcomes. For example, Kao and Brainard (2006) estimated pitch by measuring the F0 of 50-200 ms segments of harmonic syllables that lacked FM. This approach, while limited to brief segments of song (i.e., segments that are harmonic and low in FM) may provide more sensitive measurement of variation in pitch than the whole syllable measures provided by SA + . We considered the possibility that LMAN ablation may have a greater effect on pitch when syllable structure is simple, harmonic, and low in $\mathrm{FM}$ (SA + assesses the pitch of such syllables by measuring the F0, similar to the approach used by Kao and Brainard, 2006). However, a post hoc analysis of our dataset revealed that acoustically complex syllables (e.g., where syllable structure varied between harmonic and nonharmonic segments, and/or low and high FM segments) were the syllable type most likely to show reduced variation in pitch following LMAN ablation.

Another differing factor is our use of IQR to measure spectral dispersion, whereas Kao and Brainard (2006) used CV. Although the manual selection of syllable segments in Kao and Brainard (2006) limited the sample size (range of 16-90 per syllable segment), this procedure likely produced a more homogeneous dataset that could take advantage of the greater sensitivity of the CV. As shown in supplemental Material 1 (available at www. jneurosci.org), we used IQR to measure dispersion because there were often outliers in the thousands of whole syllables that we measured in each bird. IQR is largely unaffected by the presence of outliers, but in excluding the upper and lower 25\% of values some sensitivity to subtle changes in dispersion may be lost.

Another difference between studies is our use of a group of surgical control birds $(n=10)$ that matched the size of the LMAN ablation group $(n=10)$. The Kao and Brainard (2006) study compared $n=5$ birds with LMAN ablation against a hybrid control group that consisted of $n=2$ birds that were ablation misses and $n=3$ birds that were age-matched but received no surgical experience. If control surgery has an effect on variation in syllable pitch (as our findings suggest), differences in the constitution of control groups in the two studies might also have influenced the different outcomes.

It should be noted that Kao et al. (2005) presented electrophysiological findings that clearly support an LMAN influence on the dispersion and central tendency of syllable pitch. For example, they showed that microstimulation of LMAN during singing increased the dispersion and altered the central tendency of the pitch of brief syllable segments that were harmonic and lacked
FM. Interestingly, the effect on central tendency varied as a function of stimulation site, with dorsal sites decreasing, and ventral sites increasing, the central tendency of pitch. These findings show that under conditions of direct electrical stimulation LMAN has the capacity to increase the dispersion and alter the central tendency of syllable pitch.

While we found no effect of LMAN ablation on the central tendency of any spectral feature, observing such an effect would presumably require selective ablation of either the dorsal or ventral portions of LMAN. The afferent and efferent connectivity of LMAN shows a high degree of topographic organization (Johnson et al., 1995; Vates and Nottebohm, 1995; Iyengar et al., 1999), meaning that the anatomical substrate for selective effects on vocal output exists. Therefore, the ability to shift the central tendency of pitch may be a key aspect of LMAN functionality, as suggested recently by the findings of Andalman and Fee (2009) (see below).

With respect to the dispersion of pitch, we do not rule out the possibility that LMAN activity can produce a small but significant increase in the dispersion of syllable pitch [as detected under the conditions of Kao and Brainard (2006), or perhaps with a larger sample size under our conditions]. However, the pattern of statistical significance that we obtained suggests that the influence of LMAN activity on the dispersion of pitch is not as robust as the effect of LMAN activity on the dispersion of the other measured spectral (FM, entropy, pitch goodness) and temporal (syllable and intersyllable duration) features.

\section{Longitudinal comparisons suggest that LMAN contribution to the dispersion of syllable pitch is small}

The view that LMAN ablation produces a small (but potentially measureable and significant) decrease in the dispersion of syllable pitch finds support in (but is not proven by) our longitudinal comparisons between the juvenile subsong and the learned adult song of individual birds. That is, we compared the range of spectral dispersion present during subsong (when singing is driven primarily by the AFP) to the range of spectral dispersion present in adult song (when singing is driven by both VMP and AFP). While variation in syllable pitch was present during subsong, the range of dispersion was small, and syllable pitch was the only measured spectral feature that was significantly less variable during subsong than during adult song (Fig. 5). This result (which is similar to recent findings by Bottjer and Altenau, 2010) points to a role for the VMP in the development of variation in syllable pitch.

However, it is important to note that the broad dispersion of syllable pitch in adult song is due to the presence of several learned syllables, each with a distinct central tendency of pitch (Fig. 5A, see gray syllable clusters). It is therefore unclear whether the wide variation in the central tendency of the pitch of adult song syllables is inculcated gradually during juvenile song learning via recursive interaction with the AFP, whether there are developmental mechanisms of variation inherent to the VMP, or whether the vocal organ (syrinx) itself undergoes developmental changes that expand the range of pitch.

Recent data suggest that recursive interaction between the AFP and other neural pathways gradually shifts the central tendency of syllable pitch. Andalman and Fee (2009) used disrupted auditory feedback to induce young adult birds to gradually shift (over the course of several days) the pitch of harmonic song syllables and found that the AFP contributed small stepwise shifts in pitch that over time accumulated within other vocal pathways (presumably the VMP). That is, at any given point in time, the 
AFP contribution to syllable pitch was small, but several days of interaction with the AFP produced large shifts in the central tendency of syllable pitch.

One hypothesis we can formulate from these observations is that the range of dispersion generated by the AFP within a particular acoustic dimension may limit the ability of the AFP to influence central tendency within that dimension. For example, our observation that the AFP generates a comparatively small range of dispersion in syllable pitch may explain why Andalman and Fee (2009) observed small, gradual stepwise shifts in the central tendency of syllable pitch. Given our finding that AFP activity appears to generate a more robust dispersion in several other spectral (FM, entropy, pitch goodness) and temporal (syllable duration, intersyllable duration) features, it would be of future interest to determine whether birds could be induced to make large, rapid shifts in the central tendency of any of these syllable features.

\section{References}

Andalman AS, Fee MS (2009) A basal ganglia-forebrain circuit in the songbird biases motor output to avoid vocal errors. Proc Natl Acad Sci U S A 106:12518-12523.

Aronov D, Andalman AS, Fee MS (2008) A specialized forebrain circuit for vocal babbling in the juvenile songbird. Science 320:630-634.

Bottjer SW, Altenau B (2010) Parallel pathways for vocal learning in basal ganglia of songbirds. Nat Neurosci 13:153-155.

Bottjer SW, Miesner EA, Arnold AP (1984) Forebrain lesions disrupt development but not maintenance of song in passerine birds. Science 224:901-903.

Bottjer SW, Glaessner SL, Arnold AP (1985) Ontogeny of brain nuclei controlling song learning and behavior in zebra finches. J Neurosci 5:1556-1562.

Brainard MS (2004) Contributions of the anterior forebrain pathway to vocal plasticity. Ann N Y Acad Sci 1016:377-394.

Brainard MS, Doupe AJ (2000) Interruption of a basal ganglia-forebrain circuit prevents plasticity of learned vocalizations. Nature 404:762-766.

Cynx J, Shapiro M (1986) Perception of missing fundamental by a species of songbird (Sturnus vulgaris). J Comp Psychol 100:356-360.

Cynx J, Williams H, Nottebohm F (1990) Timbre discrimination in zebra finch (Taeniopygia guttata) song syllables. J Comp Psychol 104:303-308.

Foster EF, Bottjer SW (1998) Axonal connections of the high vocal center and surrounding cortical regions in juvenile and adult male zebra finches. J Comp Neurol 397:118-138.

Herrmann K, Arnold AP (1991) The development of afferent projections to the robust archistriatal nucleus in male zebra finches: a quantitative electron microscopic study. J Neurosci 11:2063-2074.

Iyengar S, Viswanathan SS, Bottjer SW (1999) Development of topography within song control circuitry of zebra finches during the sensitive period for song learning. J Neurosci 19:6037-6057.
Johnson F, Bottjer SW (1992) Growth and regression of thalamic efferents in the song-control system of male zebra finches. J Comp Neurol 326:442-450.

Johnson F, Sablan MM, Bottjer SW (1995) Topographic organization of a forebrain pathway involved with vocal learning in zebra finches. J Comp Neurol 358:260-278.

Johnson F, Soderstrom K, Whitney O (2002) Quantifying song bout production during zebra finch sensory-motor learning suggests a sensitive period for vocal practice. Behav Brain Res 131:57-65.

Kao MH, Brainard MS (2006) Lesions of an avian basal ganglia circuit prevent context-dependent changes to song variability. J Neurophysiol 96:1441-1455.

Kao MH, Doupe AJ, Brainard MS (2005) Contributions of an avian basal ganglia-forebrain circuit to real-time modulation of song. Nature 433:638-643.

Konishi M, Akutagawa E (1985) Neuronal growth, atrophy and death in a sexually dimorphic song nucleus in the zebra finch brain. Nature 315:145-147.

Lohr B, Dooling RJ (1998) Detection of changes in timbre and harmonicity in complex sounds by zebra finches (Taeniopygia guttata) and budgerigars (Melopsittacus undulatus). J Comp Psychol 112:36-47.

Lohr B, Dooling RJ, Bartone S (2006) The discrimination of temporal fine structure in call-like harmonic sounds by birds. J Comp Psychol 120:239-251.

Mooney R (1992) Synaptic basis for developmental plasticity in a birdsong nucleus. J Neurosci 12:2464-2477.

Mooney R (2009) Neurobiology of song learning. Curr Opin Neurobiol 19:654-660.

Mooney R, Konishi M (1991) Two distinct inputs to an avian song nucleus activate different glutamate receptor subtypes on individual neurons. Proc Natl Acad Sci U S A 88:4075-4079.

Nordeen KW, Nordeen EJ (1988) Projection neurons within a vocal motor pathway are born during song learning in zebra finches. Nature 334:149-151.

Simpson HB, Vicario DS (1990) Brain pathways for learned and unlearned vocalizations differ in zebra finches. J Neurosci 10:1541-1556.

Tchernichovski O, Nottebohm F, Ho CE, Pesaran B, Mitra PP (2000) A procedure for an automated measurement of song similarity. Anim Behav 59:1167-1176.

Tchernichovski O, Mitra PP, Lints T, Nottebohm F (2001) Dynamics of the vocal imitation process: how a zebra finch learns its song. Science 291:2564-2569.

Thompson JA, Wu W, Bertram R, Johnson F (2007) Auditory-dependent vocal recovery in adult male zebra finches is facilitated by lesion of a forebrain pathway that includes the basal ganglia. J Neurosci 27:1230812320.

Vates GE, Nottebohm F (1995) Feedback circuitry within a song-learning pathway. Proc Natl Acad Sci U S A 92:5139-5143.

Wu W, Thompson JA, Bertram R, Johnson F (2008) A statistical method for quantifying songbird phonology and syntax. J Neurosci Methods 174: $147-154$. 